\title{
DEPRESSION AND FUNCTIONING IN RELATION TO HEALTH CARE USE IN SICKLE CELL DISEASE,1,2
}

\author{
Merida M. Grant, Ph.D., \\ University of Pittsburgh School of Medicine \\ Karen M. Gil, Ph.D., \\ University of North Carolina at Chapel Hill
}

Marnita Y. Floyd, M.A., and

University of Virginia

Mary Abrams, M.P.H.

Duke University Medical Center

\begin{abstract}
The purpose of the current study was to investigate depression and health care use in patients with sickle cell disease (SCD). Forty-four adults with SCD were interviewed and data from 43 participants, both with $(n=11)$ and without $(n=32)$ depression, were used for further analyses. Data from one potential subject were excluded on the basis of diagnosis. The full evaluation included the Structured Clinical Interview for DSM-III-R Disorders (SCID) and the Center for Epidemiologic Studies-Depression Scale (CES-D), as well as measures of psychosocial and behavioral functioning. Good between-instrument agreement was found between the self-report and interview-based measures of depression. However, the functioning data did not entirely support the use of a more stringent cutoff score on the CES-D. Findings suggest that the purpose of the evaluation should be factored into the decision-making process when determining which cutoff score should be utilized (i.e. what is the cost-benefit ratio for false-positives vs. falsenegatives). A series of hierarchical regression analyses supported the finding that disease severity alone does not explain the level of functioning displayed by patients. More importantly, the patient's perceived functioning was the best indicator of health care use within a 1-year period. Furthermore, specific interventions that target negative thinking and distorted cognitions, as well as provide psychoeducation, such as cognitive-behavioral therapy, need to be further explored within this population.
\end{abstract}

\section{INTRODUCTION}

Studies of health care and related costs estimate that at any given time between $2 \%-10 \%$ of the population meets criteria for depression. However, nearly half of those who seek treatment do not receive an intervention from a mental health care specialist but rather from

\footnotetext{
${ }^{1}$ Preparation of this manuscript was supported in part by National Institutes of Health Grant RO1 HL46953-06, by Project VI.B.2 in the Duke University-University of North Carolina Sickle Cell Center Grant P60HL2839-12, and by the University of North Carolina at Chapel Hill General Clinical Research Center Grant RR00046. This research was also supported in part by the Fetzer Institute.

${ }^{2}$ The authors acknowledge the contribution of the staff of the Duke University-University of North Carolina Sickle Cell Center including Daria Peace, Wendell Rosse, Thomas Kinney, Patricia Thomas, and Eugene Orringer.

(C) 2000 by The Society of Behavioral Medicine.

Reprint Address: M. M. Grant, Ph.D., Western Psychiatric Institute and Clinic, Department of Psychiatry, University of Pittsburgh School of Medicine, 3811 O’Hara Street, Pittsburgh, PA 15213.
} 
a primary care physician (1). Depression may often go undetected by primary care physicians (2-4). Undiagnosed, depression in a medically ill population may result in an excess of psychosocial and behavioral dysfunction that may be incorrectly attributed to medical illness. Consequently, the symptoms targeted for treatment and subsequent outcomes may be affected.

Interest in the effects of depression on functioning and treatment outcome has increased considerably in specific disease populations, such as cancer (5-7) and neurological disorders (8-10). However, other chronic illnesses have received much less attention; one such illness is sickle cell disease (SCD). Sickle cell disease affects 1 in 400 African-Americans (11) and consists of a group of genetically similar disorders characterized by the production of abnormal hemoglobin. This abnormality episodically results in the sickling of red blood cells (12). The accumulation of sickled cells in the circulatory system prevents oxygen from reaching the intended tissue or organ, which may result in tissue death and organ failure. Acute and unpredictable painful episodes are presumed to result from the ischemic tissue injury. In general, SCD is a serious and painful disease with no cure at this time. However, prevention and maintenance treatment may allow patients to cope more effectively with the disease (13).

Differences between SCD and previously studied diseases may be categorized into two areas: (a) disease characteristics and (b) demographics. In terms of disease characteristics, SCD has both chronic and acute elements. The chronic and unpredictable painful episodes that accompany SCD, as well as serious acute and chronic complications (e.g. stroke, acute chest syndrome, etc.) have an onset that begins in early childhood. This differentiates SCD from a number of illnesses that are either acute in onset and brief in duration, or are chronic but have a much later onset.

Demographically, SCD in the United States occurs almost exclusively among AfricanAmericans (13). Several studies have documented significant differences between AfricanAmericans and non-African-Americans in the prevalence of depression and subsequent treatment-seeking behavior $(14,15)$.

\section{Depression and SCD}

Until recently, studies of adults with SCD and psychosocial functioning have focused primarily on either nonspecific psychological distress (16-18) or depressive symptoms (19), using measures which were either not specific to depression or not standardized for a medically ill population (i.e. cutoff scores were not adjusted to account for increases in somatic symptoms associated with the disease). Higher rates of psychological distress (estimated between 56\% and 77\%) (16-18) have been reported in adults with SCD compared to other outpatient primary care populations (estimated between $11 \%$ and 36\%) (20).

However, these studies may actually have reported the prevalence of general distress and not depression, or more likely, prevalence rates which were inflated by somatic symptoms.

A recent study from our research program (21) examined the prevalence of depressive symptomatology in patients with SCD using the Center for Epidemiologic StudiesDepression Scale (CES-D) (22). The CES-D is a widely-used screening measure of depression that has been validated recently for use in patients with some medical illnesses $(7,23,24)$. In this study, $43 \%$ of the 440 patients studied were found to have significant levels of depressive symptomatology using the standard, traditional cutoff score. This percentage dropped substantially to only $18 \%$ when the more stringent cutoff score recommended for those with medical illness (25) was used. Thus, a large percentage of individuals (25\%) have unexplained symptoms that may be attributable to either the disease process or to depression. We concluded from these results that future studies were needed to 
determine the most valid cutoff score on self-report measures of depression in patients with SCD.

\section{Purpose of the Present Study}

The primary purpose of the present study was to evaluate a range of cutoff scores on the CES-D by comparing responses from the self-report measure to diagnoses determined through structured clinical interviews, thus examining the sensitivity and specificity of the CES-D. The purpose of this analysis is to pragmatically examine the consequences for researchers and clinicians interested in SCD in choosing a particular cutoff score on a selfreport measure of depressive symptoms.

We thought it useful to determine the appropriate cutoff on the CES-D because it is brief, easily administered during a clinical visit, and is widely accepted as a valid screening measure of depression. The Structured Clinical Interview for DSM diagnoses (SCID) (26) was used as the basis for comparison to determine sensitivity and specificity, since it has been generally assumed that diagnostic interviews are the "gold standard" for determining the presence of depression (27). We also included measures of psychosocial and behavioral functioning to determine external validity (i.e. are different cutoff scores associated with significant differences in actual functioning). Moreover, we investigated the relationship of depression with health care use.

The specific hypotheses of the present study were: (a) that a more stringent cutoff score on a self-report measure of depressive symptoms would increase accuracy in the detection of depression when compared with the standard score for this measure, (b) the adjusted cutoff score would increase the efficacy of the measure to discriminate between functionally impaired and nonimpaired patients, and (c) that depression would contribute independent and significant variance in psychosocial and behavioral functioning beyond that attributable to the disease process associated with SCD. In contrast to previous studies of patients with $\operatorname{SCD}(28,29)$ in which psychological distress and depression were conceptualized as outcome variables, this study examined depression as a predictor of psychosocial and behavioral functioning.

\section{METHOD}

\section{Participants}

Forty-four patients recruited from the Duke University-University of North Carolina Comprehensive Sickle Cell Center were interviewed and data from 43 participants were used for further analyses. Data from one potential subject were excluded on the basis of diagnosis (i.e. patient met criteria for bipolar disorder which was part of the exclusionary criteria). The study consisted of 18 males and 25 females, all of whom were AfricanAmerican. Depressed and nondepressed subjects (based upon the clinical interview) did not differ significantly demographically. The mean educational level for those depressed versus nondepressed was 13.9 years $(S D=2.4)$ versus 13.1 years $(S D=1.4)$, and the mean age for depressed versus nondepressed was 34.8 years $(S D=7.5)$ versus 35.1 years $(S D=10.9)$. Forty percent of the participants who were not depressed were single, compared to $14 \%$ of the depressed participants. Twelve percent of the participants who met criteria for depression were unemployed, compared with $28 \%$ of the nondepressed participants.

\section{Design and Procedures}

In this cross-sectional within-group study, patients were contacted either by telephone or during clinic visits and asked to participate in an assessment of the health and functioning of patients with SCD. Consent was then obtained, followed by an interview to obtain 
demographic, disease severity data. The self-report measures of depression and psychosocial and behavioral functioning were then completed, followed by the diagnostic interview. The self-reported depression measure was completed prior to the diagnostic interview to prevent undue influence by the interview process and assure that the interviewer was blind to the patient's self-assessment prior to the diagnostic interview.

\section{Collateral Information and Follow-Up}

To address the possible influence of affect on memory (i.e. depressed patients reporting an increased number of negative events in relation to their depressed mood), collateral data were collected from informants including either a spouse, parent, or close friend (30). These data were elicited regarding the patient's psychosocial and behavioral functioning. At the time of the initial contact, the name of a significant other or close friend was solicited and a telephone interview was arranged with the confidant. Following the initial assessment, patients were provided self-monitoring records to assess health care use and disease-specific functioning over a 6-week period in order to address the difference in time frame between the health care use and psychosocial and general functioning measures.

\section{MEASURES}

\section{Depression}

The Structured Clinical Interview for DSM-III-R (SCID) (26) is a semistructured interview designed for use with either patient (SCID-P) or nonpatient (SCID-NP) populations to derive Diagnostic and Statistical Manual-III-Revised (DSM-III-R) diagnoses by trained clinicians. ${ }^{3}$ Individual items are rated as present, sub-threshold, clearly not present, or there is insufficient information provided to make the decision. For the current study, the SCIDNP (which is intended for use in research in nonpatient applications) was administered by an advanced clinical psychology graduate student (M.G.) trained in the use of the SCID. Each diagnostic interview was either audiotaped or videotaped. Tapes were reviewed and discussed with a Ph.D. level clinician (K.G.) to resolve diagnostic issues. Only the sections of the interview on affective disorders were used for diagnoses and assessed both current disorders (within the past month) and lifetime history. The sections on bipolar disorder and psychotic disorder were administered for exclusionary purposes. Data from the SCID are by definition categorical and were analyzed as depressed and nondepressed. ${ }^{4}$

A multisite study of test-retest reliability of the SCID examining current diagnoses of major depression and dysthymia based on $K$ revealed values of .64 and .40, respectively (31). These values indicate moderate to low agreement but were comparable to those found with other structured diagnostic instruments, such as the Diagnostic Interview Schedule (32). Other prior studies of interrater reliability for the SCID $(33,34)$ which have used the aforementioned procedures have found good overall agreement $(K=.74)$ and $(K=.72)$ and significantly better agreement on the diagnostic category of major depression $(K=.93)$.

Interrater Reliability-An independent rater (M.F.), an advanced graduate student in psychology trained in the use of the SCID, reviewed 20\% of the interviews. Interviews were randomly selected for review (i.e. based on a random numbers table). The coefficient $K$ was

\footnotetext{
${ }^{3}$ Although the SCID for DSM-III-R (27) was utilized for this study, criteria for both major depressive disorder and dysthymic disorder remained the same for Diagnostic and Statistical Manual-IV (DSM-IV) and subsequently findings are applicable to DSM-IV defined depression and will heretofore be addressed as DSM-IV (28) defined depression.

${ }^{4}$ Due to the limited sample size, patients who met criteria for "Depression, Not Otherwise Specified (NOS)" were included in the analyses. Depression, NOS criteria included reporting at least one "A criteria" symptom such as dysphoric mood or anhedonia and no more than three "B criteria" symptoms for a consecutive 2-week period. A diagnosis of major depression requires at least five "B criteria" symptoms.
} 
subsequently calculated to determine interrater reliability for the diagnostic interview. Only one diagnostic disagreement occurred and the tape was reviewed and resolution was obtained. Good overall agreement was found $(K=.84)$.

\section{Depressive Symptoms}

The Center for Epidemiologic Studies Depression Scale (CES-D) (22) is a 20-item measure, developed to detect symptoms of depression in the general population. Each item is scored from 0 (almost never) to 3 (almost always). The measure assesses depressive symptoms reported over a 1-week period. Total scores may range from 0 to 60. CES-D items were derived from a compilation of items from other well-validated depression scales. Four factors have been derived from the CES-D (e.g. dysphoria, positive affect, and somatic and interpersonal factors), with most of the emphasis on dysphoric mood. A cutoff score of 16 is typically used to discriminate between depressed and nondepressed mood. The validity and reliability of the CES-D are well established and generalize across demographic subgroups $(22,35)$. Items from the CES-D were summed and a total score reported. The scores were analyzed both as continuous data (i.e. as a measure of severity) and as categorical data (i.e. depressed or nondepressed) with higher scores indicating increased severity of depression.

\section{Functioning}

Psychosocial-The Social Adjustment Scale-Self Report (SAS-SR) (36) is a self-report measure of social impairment which consists of 42 questions which measure performance in six areas of functioning: housework, work outside the home or student-related activities; social and leisure activity; extended family relations; and marital role as a spouse, parent, and family member. The measure assesses functioning within a 2-week time frame. Questions are rated on a 5-point scale from 1 to 5, with higher scores indicating greater impairment.

A test of concurrent validity of the SAS-SR (based on a sample of 482 respondents from a community sample 292 psychiatric outpatients) indicated the ability to discriminate between acute depressives, alcoholics, schizophrenics, and nondepressed respondents. Acceptable internal consistency (mean alpha coefficient $=.74$ ) and moderate test-retest reliability (mean coefficient $=.80$ ) have also been established (37).

Behavioral-General behavioral functioning was assessed with the Symptom Impact Profile (SIP) (38). The SIP is regarded as a standard measure of functional status and quality of life. The 12 SIP subscales include ambulation, mobility, body care, movement, social interaction, communication, emotional behavior, alertness, eating, work, sleep, rest, and household management and are used to derive general functioning data. Respondents were asked to check each item that described their behavior and related to their health status on a given day. Responses were summed and a composite score computed. SIP scores may range between 0 and 100 with higher scores indicating more impaired functioning.

Convergent validity for the SIP with other well-established functional status measures such as the Arthritis Impact Measurement Scale (AIMS), the Functional Status Index, and the Health Assessment Questionnaire are high (.87, .73, and .78, respectively). Good discriminant validity was also found when comparing the SIP with measures of pain, and psychological and clinical measures.

Disease Specific-Functioning specifically related to SCD was assessed with the Structured Pain Interview (39). The interview assesses three pain-related variables: pain status, activity reduction, and self-reported health care utilization during the time period consisting of the 12 months prior to the interview. Painful episodes are defined as pain 
which cannot be attributed to any cause other than vaso-occlusion secondary to SCD. Pain status was assessed based upon the severity, frequency, and duration of painful episodes experienced. Severity was determined by a rating scale ranging from 0 to 10 , with zero equal to no pain and 10 equal to pain as bad as it can be. Reductions in activity were assessed using a visual analogue rating scale which ranges from $0 \%$ (do not cut back at all) to $100 \%$ (cut back completely). Activity reduction was reported as the mean percentage reduction ( $0 \%$ to $100 \%)$ in the amount of time spent engaging in either work, socializing, or household chores due to SCD crisis. Health care use was determined based upon the total number of emergency room visits, hospitalizations, and phone calls/visits to physicians.

A prior study of the reliability and validity of the Structured Pain Interview utilized a 2week self-monitoring record (29). Significant Pearson product-moment correlations were reported for pain ratings, $r(41)=.36, p<.02$, and health care contacts, $r(41)=.59, p<$. 0001. Patient-reported health care use was also compared with patient medical records. Comparisons of patientreported contacts and documented medical records also indicated significant correlations, $r(88)=.31, p<.003$. Interrater reliability for the Structured Pain Interview was determined in a separate study (40) comparing reports from adolescents with parent reports. Correlations for pain intensity, pain duration, emergency room visits, number of hospitalizations, and number of physician calls/visits were all significant (Pearson $r$ s were reported between .42-.92). Thus, no additional reliability or validity data were collected as part of the current study.

\section{Disease Severity}

No standardized measure has been developed to assess the severity of SCD. However, there are two measures which allow for the quantification of level of severity: phenotype and number of complications $(28,29)$. Phenotype is determined by hemoglobin electrophoresis. Although there is variability within type, Sickle Cell Anemia (SS) is thought to be the most severe (12). The total number of complications (e.g. leg ulcers, renal complications, aseptic necrosis, ocular complications, right upper quadrant syndrome, skeletal and joint pain, swelling of hands/feet, acute anemia, sepsis/febrile, seizures, strokes, acute chest syndrome, and priapism) were assessed by the Structured Pain Interview.

\section{Demographics}

A brief assessment of demographic information was conducted to collect data on age, sex, marital status, level of education, and socioeconomic status.

\section{RESULTS}

Three sets of analyses were performed. The first set of analyses examined the sensitivity and specificity of the CES-D in comparison with the diagnostic interview using logistic regression to investigate the use of different cutoff scores within this specific population (SCD). The second set of analyses examined between-group differences using $t$-tests (depressed versus nondepressed) by functioning (i.e. group classification determined by adjusted and nonadjusted cutoff score). The third set of analyses were hierarchical regression analyses designed to determine the amount of variance in functioning and health care use explained by self-reported depression, after controlling for demographic and disease variables.

Based upon the SCID, 25.6\% (11/43) of the participants met criteria for depression. Means and standard deviations for all measures by SCID diagnostic category are presented in Table 1. Significant between-group differences on severity of depression on the CES-D and in functioning (both psychosocial and behavioral) were found. Although depressed patients 
reported a greater number of painful episodes, emergency room visits, and acute complications, these findings did not reach statistical significance.

\section{Sensitivity and Specificity Data}

Sensitivity and specificity of the CES-D were examined through comparison with the SCID, to determine whether an increase in the cutoff score would improve the ability to detect depression in patients with SCD. A logistic regression was performed using the SCID diagnosis as the binomial dependent variable and the CES-D score as the independent variable. A classification table that generated sensitivity and specificity values was subsequently created. The raw probabilities from the table were converted into cutoff scores using the formula, $X=[\ln (1 / p-1)-$ alpha $] /$ beta, where $p$ is the probability value, alpha is the intercept parameter estimate, and beta is the parameter estimate for the CES-D. A range of consecutive cutoff scores between the traditional score of 16 and the proposed adjusted cutoff score of 27 were examined (see Table 2). No specific score derived provided an optimal false positive and false negative value that distinguished it entirely. For example, the traditional cutoff score of 16 provides good sensitivity (i.e. $81.8 \%$ ) and excellent specificity (i.e. $90.6 \%$ ) but would falsely identify $25 \%$ of patients examined as depressed. Table 2 further illustrates these findings. There were six cases where disagreement was found using the cutoff score of 16 . Most of these disagreements were false positives, where the diagnostic interview classified the patient as nondepressed but the patient met criteria for depression by self-report. The CES-D scores for the false positives ranged from 16 to 24 . In contrast, the proposed adjusted cutoff score of 27 would eliminate false positives entirely but would increase the percent of false negatives to $13.5 \%$. Cutoff scores between 17 and 26 tend to provide less sensitivity than the original score but result in improved specificity.

\section{Variability in Functioning Based Upon Different CES-D Cutoff Scores}

Differences in functioning by diagnostic category as assessed by the CES-D, using either the traditional or adjusted cutoff scores, were determined through the use of $t$-tests. The two groups (i.e. those above and below the cutoff score on the CES-D) were compared on a series of general behavioral, psychosocial, and disease-specific functioning variables. These data are presented in Table 3. Significant differences in general behavioral and psychosocial functioning were found for the traditional cutoff score but not for the proposed adjusted score. However, no significant differences were found for disease-specific functioning for either cutoff score. Further examination of Table 3 indicates that the increase in cutoff score results in higher mean scores on almost all of the functioning variables for the nondepressed group, while the means for the depressed group remained relatively stable.

\section{Hierarchical Analyses of Depression and Functioning}

The functioning data were subsequently analyzed using hierarchical regression analyses to test several models. Each hierarchical model entered an independent variable or set of variables into the equation in individual steps according to a predetermined, theoretically driven hierarchy. Step one included demographic variables (e.g. age, sex), step two included disease severity (e.g. phenotype, number of complications), and step three included level of depressive symptomatology based upon the CES-D.

Table 4 indicates that the full hierarchical models tested for behavioral functioning, $F(5,29)$ $=5.74, p<.0013$, and psychosocial functioning, $F(5,41)=11.21, p<.0001$, were significant. Individual factors that predicted behavioral functioning included sex (i.e. men reported more impaired functioning than women), the number of complications experienced (i.e. more complications were associated with more impaired functioning), and increased severity of depression as assessed by the CES-D (see Table 4). The number of complications reported accounted for $22 \%$ of the variance in behavioral functioning, while depression 
accounted for $28 \%$. Depression was the only variable that predicted psychosocial functioning as assessed by the SAS-SR and accounted for 55\% of the variance, $F=49.96, p$ $<.0001$. Each of the full models predicting functioning were significant, but age and SCD type did not predict functioning in either model.

The overall model of disease-specific functioning on the Structured Pain Interview (again, see Table 4) examining reductions in time spent on chores/household tasks was also significant and accounted for $27 \%$ of the variance, $F(5,40)=2.53, p<.05$. In addition, effects for both depression (i.e. increased severity) and sex (i.e. men reported more impaired functioning than women) were also found in that model. Again, neither age nor SCD severity expressed as either type or number of complications were significant. The analyses examining reductions in work and social activity were not significant.

An examination of the relationship between depression and health care use did not indicate a significant direct effect for depression (assessed by the CES-D) with the measures of health care use. Instead, emergency room use, hospitalizations, and visits to the doctor were all significantly correlated with the number of acute complications experienced $r(43)=.45, .51$, and $.51, p<.01$, respectively. Acute complications were significantly correlated with impaired behavioral functioning, $r(30)=.37, p<.04$, and behavioral functioning was positively correlated with the severity of depression as assessed by the CES-D, $r(30)=.53, p$ $<.0025$.

\section{Collateral Data}

Fifty-six percent (24/43) of the informants contacted provided information (Pearson rs for the SIP and SAS-SR were $r=.52, p<.01$, and $r=.41, p<.05$, respectively. Significant differences were not found between participants and their informants on any of the variables, although participants generally reported more frequent episodes, 10.12 per year (12.68) versus 8.25 (10.62), and greater duration of pain, 3.23 days (3.79) versus 2.29 days (1.69); whereas informants generally reported slightly greater impairment, $70.83 \%$ reduction (22.39) versus $65.05 \%$ reduction (25.80) for household chores, and $67.08 \%$ reduction (24.39) versus $65.41 \%$ reduction (28.41) for social functioning.

\section{Follow-Up Data}

Completed health care self-monitoring records were returned by $37 \%(16 / 43)$ of the participants. A positive and significant relationship was found between the initial evaluation and total health care contacts reported $(r=.55, p<.04)$.

\section{DISCUSSION}

Many individuals with sickle cell disease are well-adjusted psychologically despite the severity of their medical illness. However, findings from the present study along with those of prior studies (21) indicate that depression can be a problem for many patients. The incidence of depression in adults with SCD in comparison with other illness populations had previously been limited by exclusive use of self-report symptom measures. Higher rates of psychological distress (estimated between 56\% and 77\%) (16-18) were subsequently reported in adults with SCD compared to other outpatient primary care populations (estimated between 11\% and 36\%) (20). More recent studies in adults (41) have found comparable levels of psychological distress between women with SCD, breast cancer, and insulin-dependent diabetes mellitus (IDDM). In contrast, meta-analyses of developmental findings (42) indicate that children with chronic medical illnesses are at only slightly increased risk for developing depressive symptoms and do not meet clinical criteria any more often than their non-ill counterparts. Nevertheless, children with SCD do appear to be 
at greater risk for developing depression than children with other comparable disorders such as cancer, cystic fibrosis, and diabetes.

\section{Sensitivity and Specificity}

Concurrent validity of the CES-D with the SCID indicated good between-instrument agreement in identifying depressive symptomatology. However, the traditional cutoff score of 16 on the CES-D resulted in the misclassification of $12 \%$ of the sample. Most of the "misses" were false positives, wherein the patient initially reported a number of depressive symptoms and met criteria for depression on the CES-D but then subsequently did not meet criteria for depression during the interview. This concurs with the literature on depression in medically ill populations $(4,25)$, suggesting the need for higher cutoff scores to reduce the number of false positives, which may be a reflection of patients reporting symptoms of medical illness and not psychological symptoms. However, the cutoff score of 27, suggested by prior research $(4,25)$, was not entirely supported in this sample. The functioning data associated with the increased cutoff score resulted in no significant distinction between the group classified as depressed and the nondepressed group. Notably, when the cutoff score was raised to 27 on the CES-D, the nondepressed group's functioning became equally impaired as the depressed group, and more variability appears to have been introduced into the nondepressed sample as a result of the increase in the cutoff score.

One explanation for the discrepancy between the self-reported depression findings and the diagnostic interview with regard to false positives is that it may reflect a subclinical disorder of brief duration that has not persisted for the continuous 2-week period necessary to meet criteria for a diagnosis of depression in the Diagnostic and Statistical Manual (DSM) system. Another possible explanation is that patients may have acknowledged experiencing individual depressive symptoms but were uncomfortable accepting the label of "depression" which is utilized during the diagnostic interview.

If the former is true, the fact that the patient has reported significant symptomatology, regardless of its etiology, is important because findings concerning the development of depression indicate that episodes of depression tend to have an insidious onset that begins initially at subclinical levels and progressively worsen, if untreated, into full episodes (43). However, if the explanation is the latter, psychoeducation as a targeted intervention may be needed to address patient concerns regarding the label of depression and its meaning.

The findings examining between-group differences on clinically defined depression, determined by diagnostic interview and self-report, indicate greater impairment in both psychosocial and behavioral functioning in the depressed group. Similar to prior research on adjustment (18), depressed patients reported more complications and more painful episodes than the nondepressed patients, but in neither the previous study, nor the current one, did these findings reach significance.

Interestingly, it has been assumed that diagnostic interviews are the "gold standard" for determining the presence of depression, whereas self-report measures are considered less accurate. However, the current study found better agreement in terms of functioning with the self-report measure, providing additional support for the validity of the CES-D as a means of brief assessment of depression in the primary care setting.

Good psychological adjustment, a nonspecific term referring to the lack of symptoms necessary to meet criteria for an ongoing clinically defined disorder, has been found to be associated with low levels of perceived stress, infrequent use of negative thinking, and passive adherence as coping strategies and family functioning characterized by supportiveness, low conflict, and control (18). Conversely, negative thinking and passive 
adherence have been associated with greater severity of painful episodes and more frequent hospitalizations and emergency room visits (13). Both of the aforementioned studies of SCD, however, measured elements of depression but not the clinically defined syndrome that most clinicians are likely to examine.

Therefore, determining which is more important, sensitivity or specificity, may be a judgment that is best determined by the individual clinician or researcher. While the reduction of false positives (falsely categorizing a patient as depressed) has received most of the attention in the literature $(2,4,25)$, false negatives (missing someone who is indeed depressed) should be of equal concern. Additional assessment of a patient who self-reports a false positive will likely reveal the error. However, overlooking false negatives will likely result in the undertreatment or mistreatment of depression and its subsequent consequences (44).

\section{Depression and Functioning}

An examination of the relationship between depression and health care use in the form of doctor visits, emergency room use, and hospitalizations did not reveal a direct relationship. Instead, a more complex relationship between depression and health care use, influenced by degree of functional impairment was found. Specifically, similar to prior studies of health care use in patients with SCD (29), the number of doctor visits reported was predicted by disease severity (defined as a combination of both SCD type and the number of complications reported).

However, even with disease severity controlled, behavioral functioning still predicted the number of doctor visits reported and accounted for a significant proportion of the variance. While the number of doctor visits reported did not differ by diagnostic category according to the SCID interview, a functioning by group difference was found for self-reported depression. Patients who self-reported as depressed also reported twice as many doctor visits in a year as the nondepressed group. The combined factors of disease severity and functioning explained almost half of the variance in doctor visits.

In conclusion, the current study suggests that there appears to be an overemphasis placed on cutoff scores on measures of depression. However, improved agreement between self-report and interview-based measures of depression are not necessarily accompanied by improved agreement with level of functioning. As found in several prior studies $(13,29,39)$, even severity of disease alone does not explain the level of functioning displayed by a given patient. Instead, health care utilization appears to be significantly affected by perceived functioning. To the extent that depression contributes to worsening of functioning beyond that attributable to the disease process, one area of intervention which may be targeted in terms of reducing high health care utilization may be the assessment and treatment of depression in this population. However, an alternative explanation that cannot be ruled out by the current study has been suggested by McCrae and Lumley (45), suggesting that negative thinking and somatic awareness (i.e. bodily hypervigilance) may be components of the same construct, namely negative affectivity. Nevertheless, these findings would still suggest that psychological factors, and not simply disease severity, should be targeted for intervention.

Limitations of the current study include the need for behavioral observation of functioning by trained observers to rate level of functioning independently. While patients and their nominated informants generally agree on functioning, neither is an unbiased observer. This step would further clarify any differences in actual versus perceived functioning and the subsequent impact on health care utilization. Further, the addition of a control comparison group without SCD could provide baseline rates of depression and functioning in a 
comparable demographic sample. However, the inclusion of patients with SCD, both with and without depression, in the current study may partially address this issue, since all subjects were similar with regard to disease status and demographics such as race, age, and education.

A separate issue of concern is in relation to the differing time periods under assessment by the various measures in the study. Behavioral functioning and health care use are constructs that are assessed over a 12-month time frame necessarily, since as the current data reveal, outpatient samples of SCD patients do not utilize health care services or experience painful episodes very often within a 2-week period (the time frame for assessing the psychosocial measures). However, recent findings suggest that even with treatment, the median duration of a first episode of depression is 22 weeks (46). Therefore, with or without treatment, there is an expected stability of mood for a significant portion of the assessment period utilized for the assessment of health care use and functioning. However, to address this matter, a 6-week prospective follow-up of daily functioning was included to assess whether there were significant correlations between the initial evaluation of health care use and follow-up contacts reported. Although a significant relationship was found, measures with comparable assessment time frames would better address this problem.

Similarly, the use of a cross-sectional design prohibits the interpretation of the current finding of decreased functioning in depressed subjects as causal, since decreased functioning as a result of the disease process could just as well contribute to the onset of depression. However, this possibility is attenuated by the fact that, regardless of the etiology of the decreased functioning, it is associated with both increased health care use and risk of developing a more chronic course of depression.

Prior research in first-visit psychiatric patients $(47,48)$ have compared the CES-D with a semistructured interview (e.g. the Psychiatric Initial Screening for Affective Disorders [PISA]) in a Japanese sample. The comparison of alternative scoring methods, using receiver operating characteristic analysis (ROC), indicated that the traditional scoring method was most appropriate for this sample. An ROC analysis is an alternative and sometimes preferable strategy that examines the ability of a measure to distinguish signal (sensitivity) from noise (specificity) and should be considered in future studies.

A final constraint through which all findings from this work must be interpreted is the limited number of patients with depression $(n=11)$ in this sample. A larger study of depressed patients with SCD may reflect a more representative distribution of functioning and health care use and allow for more elaborate analysis, such as an ROC. The current study provides preliminary evidence of impaired functioning that should serve as the basis for a follow-up investigation that utilizes oversampling for depression to address this limitation.

In short, this work suggests future intervention studies that target clinical and subclinical symptoms of depression in patients with SCD are needed. Findings from our prior studies indicate that training in cognitive-behavioral pain coping skills leads to important benefits for patients with SCD $(49,50)$. Specifically, interventions that target coping skills, depressive cognitions, and provide psychoeducation $(39,51)$ may well prevent the onset of a pattern of chronic depression secondary to disease and further improve the quality of life for patients.

\section{References}

1. Narrow WE, Regier DA, Rae DS, Manderscheid RW, Locke BZ. Use of services by persons with mental and addictive disorders: Findings from the National Institute of Mental Health 
Epidemiological Catchment Area Program. Archives of General Psychiatry 1993;50:95-107. [PubMed: 8381266]

2. Gerber PD, Barrett J, Barrett J, et al. Recognition of depression by internists in primary: A comparison of internist and gold standard psychiatric assessments. Journal of General Internal Medicine 1988;4(1):7-13. [PubMed: 2915277]

3. Perez-Stable EJ, Miranda J, Munoz RF, Ying YW. Depression in medical outpatients: Underrecognition and misdiagnosis. Archives of Internal Medicine 1990;150(5):1083-1088. [PubMed: 2184790]

4. Schulberg HC, Saul M, McClelland M, et al. Assessing depression in primary medical and psychiatric practices. Archives of General Psychiatry 1985;42(12):1164-1170. [PubMed: 4074109]

5. Bukberg J, Holland JC. A prevalence study of depression in a cancer hospital population. Proceedings of the American Association of Cancer Research 1980:21-382.

6. Dean C. Psychiatric morbidity following mastectomy: Preoperative predictors and types of illness. Journal of Psychosomatic Research 1987;31:385-392. [PubMed: 3625592]

7. Evans DL, McCartney CF, Nemeroff CB, et al. Depression in cancer patients: Diagnostic and treatment considerations. North Carolina Medical Journal 1988;49(10):546-548. [PubMed: 3141819]

8. Finklestein S, Benowitz LI, Baldessarini RJ, et al. Mood, vegetative disturbance, and dexamethasone suppression test after stroke. Annals of Neurology 1982;12(5):463-468. [PubMed: 6960804]

9. Minden SL, Orav J, Reich P. Depression in multiple sclerosis. General Hospital Psychiatry 1987;9(6):426-434. [PubMed: 3692149]

10. Santamaria J, Tolosa E, Valles A. Parkinson's disease with depression: A possible subgroup of idiopathic parkinsonism. Neurology 1986;36(8):1130-1133. [PubMed: 3736883]

11. Concensus Conference: Newborn screening for sickle cell disease and other hemoglobinopathies. Journal of the American Medical Association 1987;258(9):1205-1209. [PubMed: 3626004]

12. Charache, S.; Lubin, B.; Reid, CD. Management and Therapy of Sickle Cell Disease. Washington, DC: U.S. Government Printing Office; 1992. NIH Publication No. 84-2117

13. Gil KM. Coping with sickle cell disease pain. Annals of Behavioral Medicine 1989;11(2):49-57.

14. Kessler RC, McGonagle KA, Zhao S, et al. Lifetime and 12-month prevalence of DSM-III-R psychiatric disorders in the United States: Results from the National Comorbidity Survey. Archives of General Psychiatry 1994;51(1):8-19. [PubMed: 8279933]

15. Somervell PD, Leaf PJ, Weissman MM, Blazer DG, Bruce ML. The prevalence of major depression in Black and White adults in five United States communities. American Journal of Epidemiology 1989;130(4):725-735. [PubMed: 2788995]

16. Barret DH, Wisotzek IE, Abel GG, et al. Assessment of psychosocial functioning of patients with sickle cell disease. Southern Medical Journal 1988;81(6):745-750. [PubMed: 3375880]

17. Damlouji NF, Kevess-Cohen R, Charache S, Georgopoulos A, Folstein MF. Social disability and psychiatric morbidity in sickle cell anemia and diabetes patients. Psychosomatics 1982;23(9):925931. [PubMed: 7146288]

18. Thompson RJ, Gil KM, Abrams MR, Phillips G. Stress, coping, and psychological adjustment of adults with sickle cell disease. Journal of Consulting and Clinical Psychology 1992;60(3):433440. [PubMed: 1619097]

19. Belgrave FZ, Molock SD. The role of depression in hospital admissions and emergency treatment of patients with sickle cell disease. Journal of the National Medical Association 1991;83(9):777781. [PubMed: 1942111]

20. Schulberg HC, Burns BJ. Mental disorders in primary care: Epidemiologic, diagnostic, and treatment research directions. General Hospital Psychiatry 1988;10(2):79-87. [PubMed: 3282988]

21. Schaeffer JW, Gil KM, Burchinal M, et al. Depression, disease severity and sickle cell disease. Journal of Behavioral Medicine 1999;22(2):115-126. [PubMed: 10374138]

22. Radloff LS. The CES-D scale: A self-report depression scale for research in the general population. Applied Psychological Measurement 1977;1(3):385-401. 
23. Minden SL, Orav J, Reich P. Depression in multiple sclerosis. General Hospital Psychiatry 1987;9(6):426-434. [PubMed: 3692149]

24. Rucker L, Frye EB, Cygan RW. Feasability and usefulness of depression screening in medical outpatients. Archives of Internal Medicine 1986;146(4):729-731. [PubMed: 3963955]

25. Zich JM, Attkisson CC, Greenfield TK. Screening for depression in primary care clinics: The CESD and the BDI. International Journal of Psychiatry in Medicine 1990;20(3):259-277. [PubMed: 2265888]

26. Spitzer RL, Williams JBW, Gibbon M, First MB. The structured clinical interview for DSM-III-R (SCID) I: History, rationale and description. Archives of General Psychiatry 1992;49:624-629. [PubMed: 1637252]

27. Malgady RG, Rogler LH, Tryon WW. Issues of validity in the Diagnostic Interview Schedule. Journal of Psychiatric Research 1992;26(1):59-67. [PubMed: 1560410]

28. Gil KM, Abrams MR, Phillips G, Keefe FJ. Sickle cell disease pain: Relation of coping strategies to adjustment. Journal of Consulting and Clinical Psychology 1989;57(6):725-731. [PubMed: 2600243]

29. Gil KM, Abrams MR, Phillips G, Williams DA. Sickle cell disease pain: 2. Predicting health care use and activity level at 9-month follow-up. Journal of Consulting and Clinical Psychology 1992;60(2):267-273. [PubMed: 1592957]

30. Blaney PH. Affect and memory: A review. Psychological Bulletin 1986;99(2):229-246. [PubMed: 3515383]

31. Williams JBW, Gibbon M, First MB, et al. The structured clinical interview for DSM-III-R (SCID). Archives of General Psychiatry 1992;49:630-636. [PubMed: 1637253]

33. Robins LN, Helzer JE, Coughran J, Ratcliff KS. National Institute of Mental Health Diagnostic Interview Schedule. Its history, characteristics, and validity. Archives of General Psychiatry 1981;38(4):381-389. [PubMed: 6260053]

34. Riskind JH, Beck AT, Berchick RJ, Brown G, Steer RA. Reliability of DSM-III diagnoses for major depression and generalized anxiety disorder using the structured clinical interview for DSMIII. Archives of General Psychiatry 1987;44(9):817-820. [PubMed: 3632255]

35. Skre I, Onstad S, Torgerson S, Kringleu E. High interrater reliability for the structured clinical interview for DSM-III-R axis I: SCID. Acta Psychiatrica Scandinavica 1991;84:167-173. [PubMed: 1950612]

36. Roberts RE. Reliability of the CES-D scale in different ethnic contexts. Psychiatry Research 1980;2:125-134. [PubMed: 6932058]

37. Weissman MM, Prusoff BA, Thompson WD, Harding PS, Myers JK. Social adjustment by selfreport in a community sample and in psychiatric outpatients. Journal of Nervous and Mental Disease 1978;166(5):317-326. [PubMed: 650195]

38. Weissman MM, Bothwell S. Assessment of social adjustment by patient self-report. Archives of General Psychiatry 1976;33:1111-1115. [PubMed: 962494]

39. Bergner M, Bobbitt RA, Carter WB, Gilson BS. The Sickness Impact Profile: Development and final revision of a health status measure. Medical Care 1981;19(8):787-805. [PubMed: 7278416]

40. Gil KM, Phillips G, Webster DA, et al. Experimental pain sensitivity and reports of negative thoughts in adults with sickle cell disease. Special Issue: Experimental pain as a model for the study of clinical pain. Behavior Therapy 1995;26(2):273-293.

41. Ohaeri JU, Campbell OB, Ilesanmil AO, Ohaeri BM. Psychosocial concerns of Nigerian women with breast and cervical cancer. Psycho-Oncology 1998;7(6):494-501. [PubMed: 9885090]

42. Bennett DS. Depression among children with chronic medical problems: A recta-analysis. Journal of Pediatric Psychology 1994;19(2):149-169. [PubMed: 8051600]

43. Keller MB, Lavori PW, Mueller TI, et al. Time to recovery, chronicity, and levels of psychopathology in major depression. A 5-year prospective follow-up of 431 subjects. Archives of General Psychiatry 1992;49(10):809-816. [PubMed: 1417434]

44. Weissman MM, Klerman GL. The chronic depressive in the community: Unrecognized and poorly treated. Comprehensive Psychiatry 1977;18(6):523-532. [PubMed: 923224] 
45. McCrae JD, Lumley MA. Health status in sickle cell disease: Examining the roles of pain coping strategies, somatic awareness, and negative affectivity. Journal of Behavioral Medicine 1998;21(1):35-55. [PubMed: 9547421]

46. Solomon DA, Keller MB, Leon AC, et al. Recovery from major depression: A 10-year prospective follow-up across multiple episodes. Archives of General Psychiatry 1997;54(11):1001-1006. [PubMed: 9366656]

47. Furakawa T, Hirai T, Kitamura T, Takahashi K. Application of the center for epidemiologic studies depression scale among first-visit psychiatric patients: A new approach to improve its performance. Journal of Affective Disorders 1997;46:1-13. [PubMed: 9387082]

48. Furakawa T, Anraku K, Hiroe T, et al. Screening for depression among first-visit psychiatric patients: Comparison of different scoring methods for the center for epidemiologic studies depression scale using receiver operating characteristic analyses. Psychiatry and Clinical Neuroscience 1997;51:71-78.

49. Gil KM, Wilson JJ, Edens JL, et al. The effects of cognitive coping skills training on coping strategies and experimental pain sensitivity in African American adults with sickle cell disease. Health Psychology 1996;15:3-10. [PubMed: 8788535]

50. Gil KM, Wilson JJ, Edens JL, et al. Cognitive coping skills training in children with sickle cell disease. International Journal of Behavioral Medicine 1997;4:365-378.

51. Wilson JJ, Gil KM, Raezer LB. Self-evaluation, coping and depressive affect in African American adults with sickle cell disease. Cognitive Therapy and Research 1997;21:443-457. 


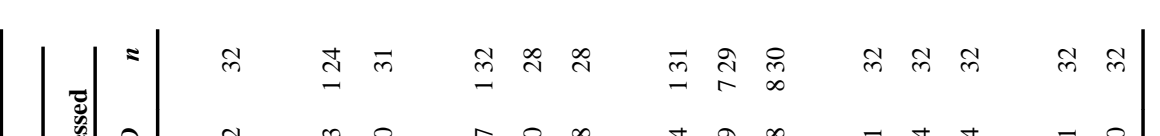

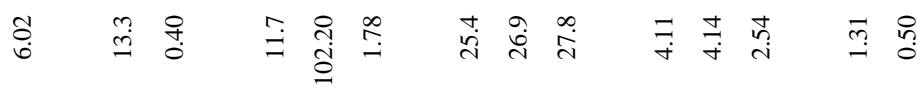

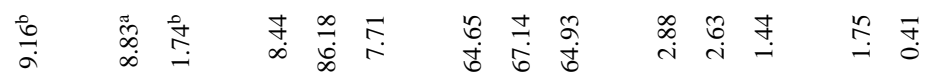

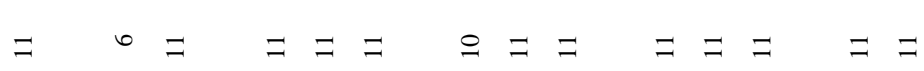

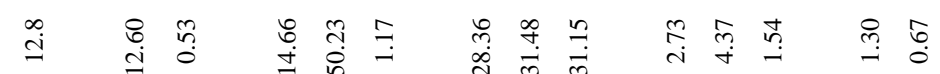

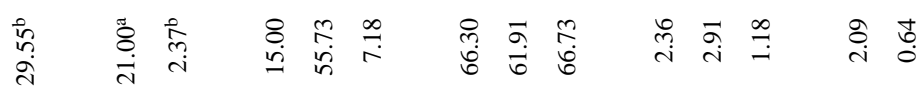

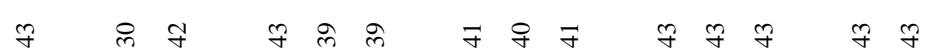

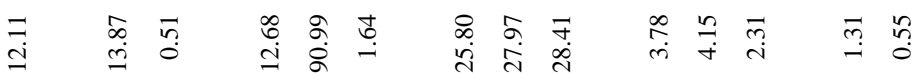

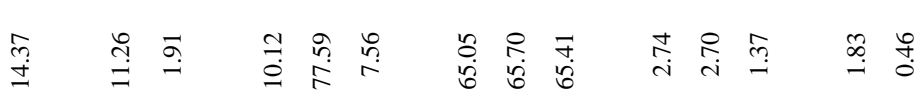


TABLE 2

Sensitivity and Specificity of the CES-D for Traditional and Adjusted Cutoff Scores

\begin{tabular}{lccrr}
\hline Score & Sensitivity $\%$ & Specificity $\%$ & False Positive $\%$ & False Negative $\%$ \\
\hline $16^{*}$ & 81.8 & 90.6 & 25.0 & 6.6 \\
17 & 72.7 & 90.6 & 27.3 & 9.4 \\
18 & 72.7 & 93.8 & 20.0 & 9.1 \\
19 & 72.7 & 93.8 & 20.0 & 9.1 \\
20 & 63.6 & 93.8 & 22.2 & 11.8 \\
21 & 63.6 & 93.8 & 22.2 & 11.8 \\
22 & 63.6 & 96.9 & 12.5 & 11.4 \\
23 & 63.6 & 96.9 & 12.5 & 11.4 \\
24 & 63.6 & 96.9 & 12.5 & 11.4 \\
25 & 54.5 & 96.9 & 14.3 & 13.9 \\
26 & 54.5 & 96.9 & 14.3 & 13.9 \\
27 & 54.5 & 100.00 & 0.0 & 13.5 \\
\hline
\end{tabular}

Note: Sensitivity and specificity are provided for each cutoff score on the CES-D between 16 and 27 in addition to the number of false positives and false negatives generated by that score in this sample. (Example: Score of 25 would only correctly detect about 55\% of cases but would rule out most noncases).

Refers to the traditional cutoff score of 16. 


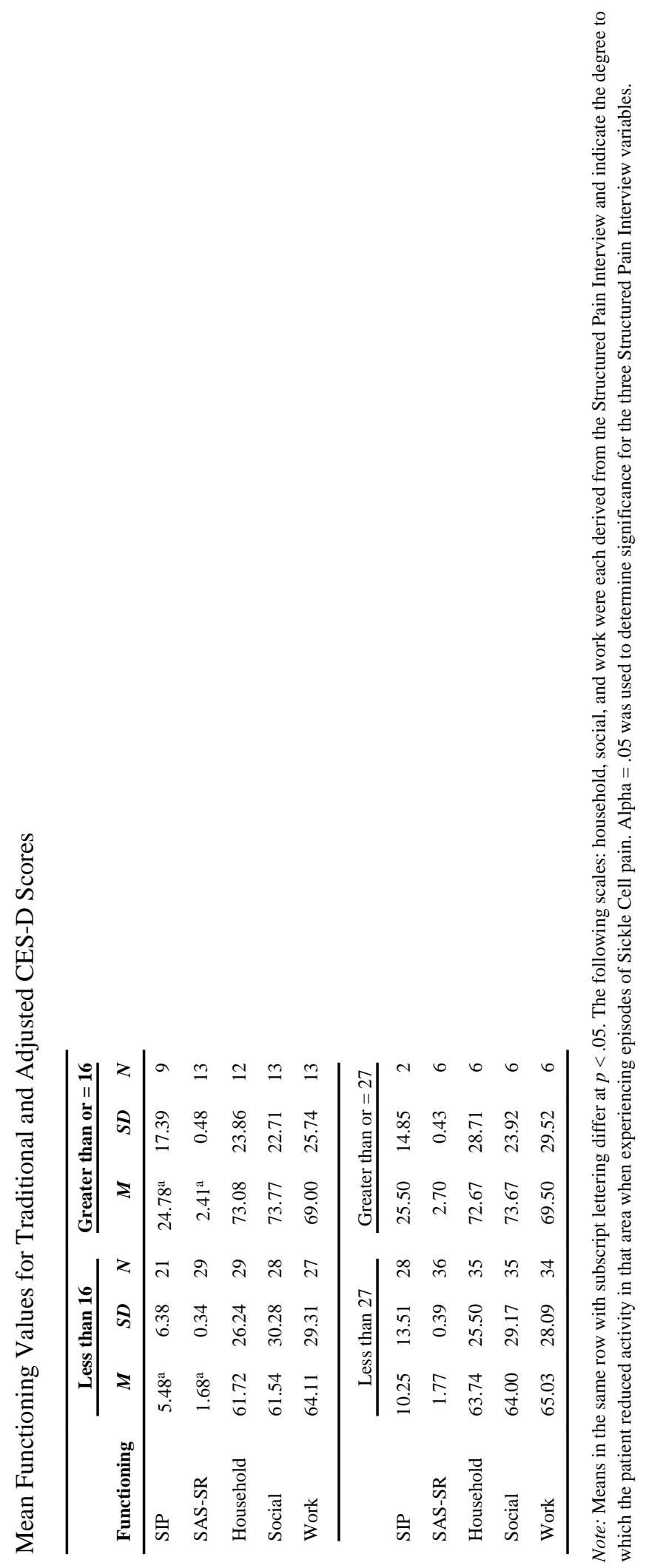




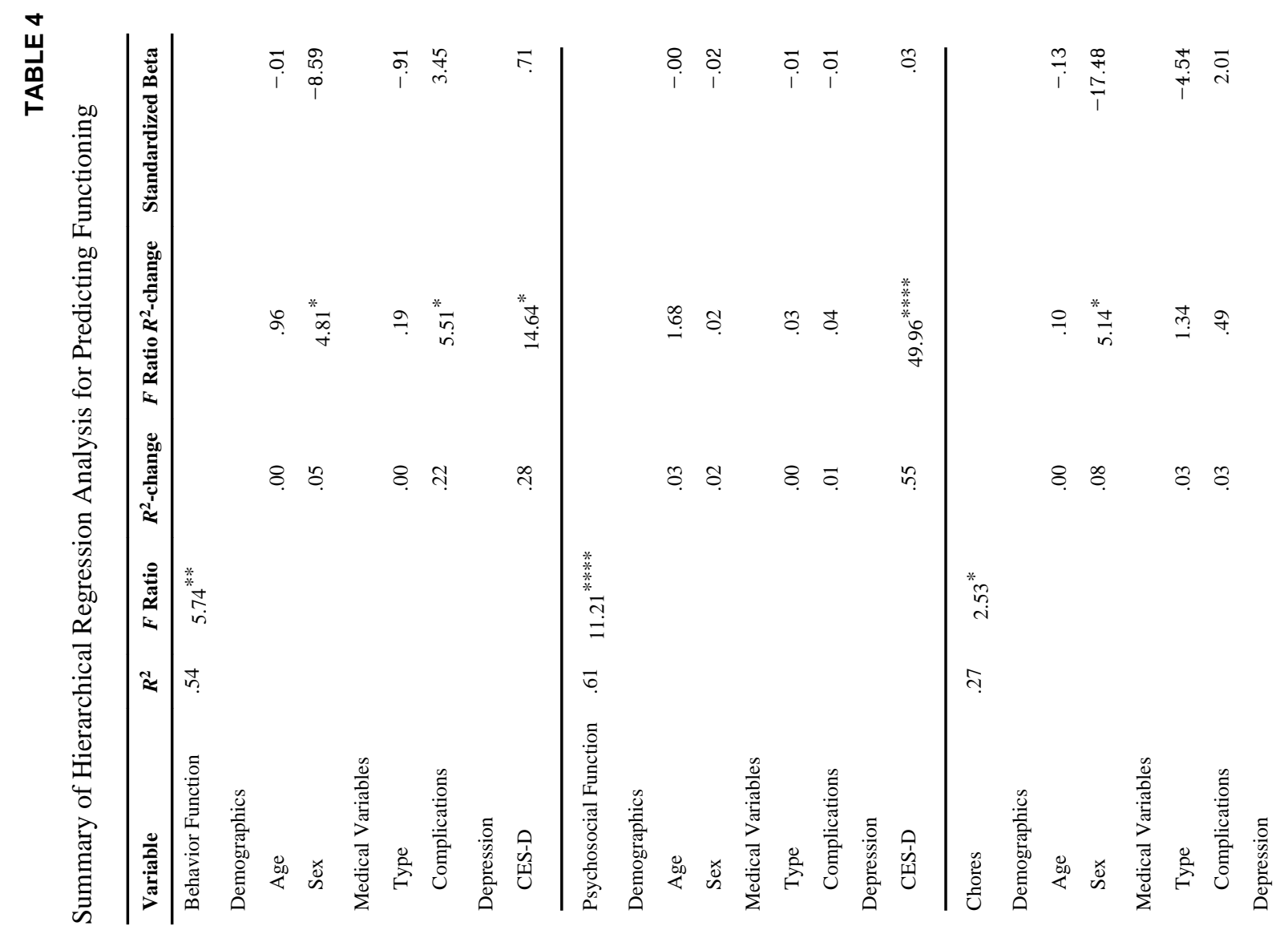

Ann Behav Med. Author manuscript; available in PMC 2011 January 10. 


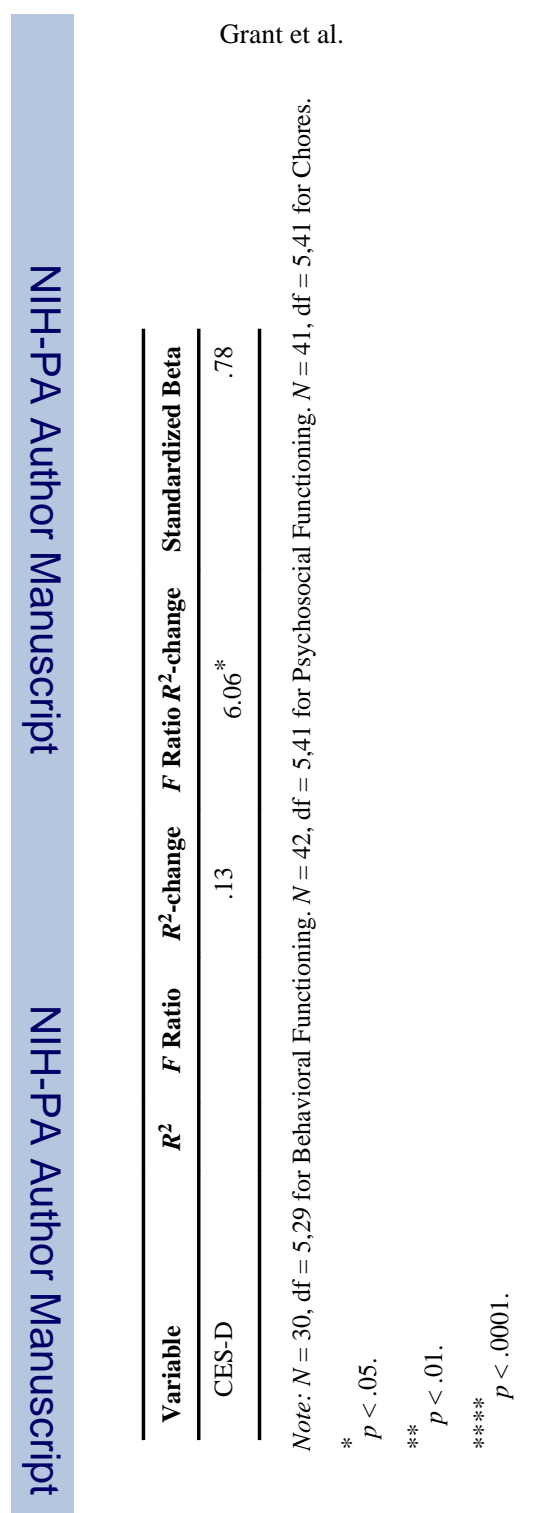

Page 19

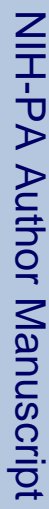

Ann Behav Med. Author manuscript; available in PMC 2011 January 10. 